\title{
Prevalence of microsatellite instability, Lynch syndrome, and PD-L1 expression in patients with Epstein-Barr virus negative gastric carcinoma with lymphoid stroma
}

SUN MI LEE ( $\sim$ drsunmi@yahoo.com )

Jeju National University Hospital https://orcid.org/0000-0003-2350-4765

\section{Sungjoon Park}

Jeju National University Hospital

\section{Research Article}

Keywords: Gastric carcinoma with lymphoid stroma, Epstein Barr Virus, microsatellite instability, Lynch syndrome, PD-L

Posted Date: January 28th, 2022

DOI: https://doi.org/10.21203/rs.3.rs-1267422/v1

License: (a) (i) This work is licensed under a Creative Commons Attribution 4.0 International License. Read Full License 


\section{Abstract \\ Purpose}

The prevalence of the Lynch syndrome and microsatellite instability (MSI) among patients with gastric carcinoma with lymphoid stroma (GCLS) is poorly understood. The present study aimed to investigate MSI and programmed death-ligand 1 (PD-L1) expression and further evaluate Lynch syndrome in patients with GCLS.

\section{Methods}

The study included 104 patients with GCLS (30 EBV-negative and 74 EBV-positive cases) who underwent surgical resection. Immunohistochemistry for mismatch repair proteins and PD-L1 (SP263), along with MSI testing, were performed. Whole-exome sequencing was performed for patients suspected of having Lynch syndrome.

\section{Results}

Compared with patients with EBV-positive GCLS, patients with EBV-negative GCLS were more likely to be older, female, and have a higher rate of MSI-high (MSI-H) phenotype incidence, with a primary tumour site predisposition to the distal stomach. They also tended to have a history of metachronous cancers and high expression of the PD-L1 protein. Fifteen (50\%) of the 30 cases were of the MSI-H phenotype with loss of MLH1 and PMS2 expression. Among them, two (6.7\%) patients were found to have Lynch syndrome with germline mutations in MLH1 and PMS2.

\section{Conclusions}

Evaluation of MSI is highly recommended in patients with EBV-negative GCLS. In patients with MSI-H tumours, selective genetic consultation and testing are required to detect Lynch syndrome. In addition, the rate of PD-L1 positivity in both EBV-negative and EBV-positive GCLS was relatively high $(13.3 \%$ and $29.7 \%$, respectively). Therefore, patients with GCLS, regardless of EBV infection, can be good candidates for treatment with immune checkpoint inhibitors.

\section{Introduction}

Lynch syndrome (LS) is an autosomal dominant cancer predisposition syndrome caused by germline pathologic variants in four mismatch repair (MMR) genes, including $M L H 1, M S H 2, M S H 6$, and PMS2. Moreover, a constitutional 3'-end deletion of EPICAM, which is immediately upstream of $M S H 2$, may cause LS through epigenetic silencing of $M S H 2$ (Kuiper et al. 2011). Patients with LS have an elevated risk of colorectal cancer (CRC; $60 \%-80 \%)$, endometrial cancer (30\%-70\%), and tumours in other organs, 
including the stomach, small bowel, pancreaticobiliary tract, urothelial tract, ovary, and skin (Lynch and de la Chapelle 1999; Bonadona et al. 2011; Watson et al. 2008). Impaired MMR systems enhance somatic mutation accumulation; thus, most LS-related cancers exhibit microsatellite instability (MSI) (Julie et al. 2008). Accordingly, evaluation of MSI using immunohistochemistry and/or polymerase chain reaction (PCR) amplification of microsatellite sequences (MSI testing) on cancer specimens is the initial step in universal screening for LS (Stoffel et al. 2015). In CRC, the MSI-high (MSI-H) phenotype is characterised by clinicopathologic features distinct from those observed in microsatellite stable (MSS) tumours, including right-sided tumour location, signet ring cell features, mucinous histology, poor differentiation, and tumour infiltrating lymphocytes (Raut et al. 2004). Among the histologic subtypes of gastric carcinomas (GCs), gastric carcinomas with lymphoid stroma (GCLS) present with distinct histologic features of poor differentiation and tumour infiltrating lymphocytes, which has been frequently observed in CRCs with the MSI-H phenotype. Therefore, evaluation of the MSI status of these tumours can be useful to determine the MSI-H phenotype and for detection of LS in patients with GC.

GCLS is a rare histologic subtype of GC, and was previously termed medullary carcinoma, lymphoepithelioma-like carcinoma, or undifferentiated carcinoma with lymphoid stroma (Carneiro et al. 2019). This histologic subtype accounts for approximately $9 \%$ of all GC cases (Murphy et al. 2009). Epstein-Barr virus (EBV) infection is commonly associated with this type of GC (Shinozaki-Ushiku et al. 2015). Approximately $80 \%$ of GCLSs are infected with EBV (Shinozaki-Ushiku et al. 2015; Min et al. 2016). Therefore, a majority of studies have focused on the clinicopathological features and survival associated with EBV-positive GCLSs. Patients with EBV-positive GCLS present with distinct clinicopathologic characteristics such as male predominance, tumour location predisposition to the proximal part of the stomach or anastomotic site, infrequent lymphatic or vascular invasion, distant metastasis, and a better prognosis compared to that of patients with conventional GC (Lee et al. 2009; Camargo et al. 2014; Park et al. 2015). Reportedly, EBV-positive GCs, including GCLS tumours, are almost exclusively associated with the MSS, but not with the MSI-H phenotype (Saito and Kono 2021). Although there is a subset of EBV-negative GCLS tumours, MSI status in these tumours has not been well investigated due to the rarity of these tumours or lack of recognition.

In this study, we investigated the MSI status in 104 GCLS cases using immunohistochemistry and MSI testing. Methylation analysis was performed to find MLH1-hypermethylation in all MSI-H tumours. Patients with suspected LS were identified after a review of the personal and family histories of the cancers and onset of age in patients with MSI-H. Whole-exome sequencing (WES) was performed on the healthy tissue samples of these patients to confirm LS-related germline mutations. In addition, we evaluated the expression of PD-L1 (SP263) in GCLS tumours.

\section{Patients And Methods}

\section{Case collection and histologic evaluation}


The study was approved by the Institutional Review Board of Jeju National University Hospital, Jeju-do, Korea. The institutional database of Jeju National University Hospital, accessed at the Department of Pathology, was reviewed, and patients diagnosed with primary GCLS, lymphoepithelioma-like carcinoma, medullary carcinoma, or undifferentiated carcinoma with lymphoid stroma between 2007 and 2018 were identified. All patients underwent radical gastrectomy with D2 lymph node dissection. Cases where the primary site of origin could not be reliably established as the stomach were excluded from the analysis. A total 154 of surgical cases were identified in this search, and all Hematoxylin \& Eosin (H\&E) stained slides were reviewed by two gastrointestinal and liver pathologists (SL and SP). Among these, 104 surgical cases were included in the study. The diagnosis of GCLS in each case was made based on the definition of GCLS provided by the World Health Organization (WHO) blue book for digestive system tumours (Carneiro et al. 2019). Histologically, GCLS is characterised by irregular sheets, trabeculae, ill-defined tubules, or syncytia of polygonal cells embedded within a prominent lymphocytic infiltrate, with intraepithelial lymphocytes. These polygonal tumour cells show indistinct cytologic borders, large nuclei with open chromatin, and prominent nucleoli. If GCs only exhibit occasional intratumoural or peritumoral lymphocytes without having a dense lymphocytic infiltrate, so-called "lymphoid stroma", cases of these tumours were not met the criteria of WHO classification and were excluded in this study. All the patients were restaged according to the tumour-node-metastasis (TNM) classification (8th edition) developed by the American Joint Committee on Cancer (AJCC) (Ajani et al. 2017). Clinical data, including demographic, treatment, and follow-up data, were obtained from the patients' medical records. Family and personal histories of cancers and age of onset were reviewed to find if patients were met under the revised Bethesda guidelines (Umar et al. 2004) and Amsterdam II criteria (Vasen et al. 1999).

\section{EBV infection status evaluated by EBV-encode RNA in situ hybridization}

Three-micrometer-thick sections were cut from each tissue block and mounted on Superfrost-Plus slides (Thermo Scientific, Waltham, MA, USA). The entire procedure was performed with a fully automatic system (BOND-MAX, Leica, Newcastle, UK) for in situ hybridisation with an EBV-encoded Ribonucleic Acid (RNA) probe (Leica, Newcastle, UK) according to the manufacturer's instructions. Tissue sections showing strong labelling in almost all tumour cell nuclei were considered positive.

\section{Immunohistochemistry for MMR proteins and PD-L1}

Immunohistochemistry was performed on representative whole tissue sections using the avidin-biotin method. The primary antibodies used were against MLH1 (mouse monoclonal antibody clone G168-728 at a dilution of 1:300; Cell Marque, CA, USA), MSH2 (mouse monoclonal antibody clone FE11 at a dilution of 1:100; Calbiochem, CA), MSH6 (mouse monoclonal antibody 44 at a dilution of 1:300; BD Transduction Laboratories, CA, USA), and PMS2 (mouse monoclonal antibody clone A16-4 at a dilution of 1: 125; BD Transduction Laboratories, CA). An automated Ventana Medical Systems stainer (Ventana Medical Systems) was used for staining according to the vendor's manual. Immunohistochemical expression of each MMR protein was considered intact if nuclear staining of neoplastic cells was detected with internal control positivity in the non-neoplastic crypt epithelium. The intensity and 
proportion of PD-L1 expression in all the immunostained tissue sections was also evaluated. Cytoplasmic and/or membranous staining of PD-L1 in tumour cells was considered positive for PD-L1 expression. Immunoreactivity was scored according to the staining intensity as follows: negative (0), weak (1+), moderate (2+), and strong (3+). The proportion of PD-L1 positive tumour cells per whole tumour cells was also evaluated. Tumours with intensity scores of $2+$ and $3+$ in regions with $\geq 10 \%$ of positive tumour cells were defined as PD-L1 positive tumours (Phillips et al. 2015; Choi et al. 2019).

\section{MSI testing}

MSI analysis was performed using multiplex PCR with five quasi-monomorphic mononucleotide repeat markers, including BAT26, D5S346, BAT 25, D17S250, and D2S123. Genomic DNA was isolated from formalin-fixed, paraffin-embedded specimens using a QIAamp DNA Mini Kit (Qiagen, Valencia, CA, USA). After PCR amplification with specific primers, the fluorescent-labelled products were subjected to fragment analysis using an ABI 3130 or 3500 Genetic Analyser (Applied Biosystems, Foster City, CA, USA). Allelic sizes were estimated using the Genemapper 4.1 (Applied Biosystems). MSI results were recorded as follows: microsatellite stable (MSS), no allele shift; MSI-L (low), one allelic shift; MSI-H (high), at least two allelic shifts. Because MSI-L and MSS tumours are known to show similar clinical features (Laiho et al. 2002), these phenotypes were both considered MSS in our study.

\section{Methylation analysis of the MLH1 promotor}

Methylation analysis of the promoter regions of MLH1 was performed on MSI-H tumour blocks using MethyLight, a methylation-specific real-time PCR-based method. The methylation status of a sample was considered positive if the methylated reference volume was $>10 \%$ (Ogino et al. 2006).

\section{Detection of germline mutations by whole exome sequencing}

For evaluation of germline gene variants in genes associated with genetic tumours of the digestive system, WES was performed on healthy samples from seven patients who were suspicious of LS according to personal and family history of cancers and MSI-H phenotype. Genomic DNA was extracted from formalin-fixed and paraffin-embedded (FFPE) healthy tissues using the Maxwell 16 FFPE Plus LEV DNA Purification Kit (Promega, Mannheim, Germany). Exome capture was performed using the Agilent SureSelect Human All Exon V5 Kit (Agilent Technologies, Santa Clara, CA, USA) according to the manufacturer's instructions. WES was performed on the Illumina HiSeq 2500 platform (Illumina, Inc., San Diego, CA, USA) according to the Illumina protocols and recommendations. Following the removal of the low-quality sequencing data, sequencing reads were mapped to the reference human genome (hg 19) using the Burrows-Wheeler Alignment aligner (version 0.7.12.) using the default options. Local realignment and base quality recalibration were performed using the Genome Analysis Toolkit (GATK version 3.8.). Single nucleotide variants (SNVs) and small insertion or deletion (INDEL) calling were analysed by MuTect2 (version I.1.7.). Copy number variant calling was conducted using CONTRA (version 2.0.8). 


\section{Statistical analysis}

Continuous variables and proportions were compared using the independent t-test and the MannWhitney Utest as appropriate. Chi-square or Fisher's exact tests were used to test possible correlations between EBV infection, MSI status, PD-L1 expression, and various clinicopathologic factors. The probability of overall survival (OS) was estimated using the Kaplan-Meier method and compared using Cox proportional hazards regression models. Statistical significance was set at $p<0.05$. All statistical analyses were performed using SPSS software (version 22.0; SPSS Inc., Chicago, IL, USA).

\section{Results}

\section{Clinicopathologic features of EBV-negative tumours}

Among 104 GCLS tumours, 74 (71.1\%) and 30 (28.5\%) were EBV-positive and EBV-negative, respectively. The clinicopathological features of EBV-negative and positive GCLSs are summarised in Table 1. Patients with EBV-negative GCLS were more likely to be older (median age, 65.5 years) and female, with a higher incidence of a history of metachronous cancers, and the distal stomach as the primary tumour site, compared with those of patients with EBV-positive GCLS. Five patients with EBV-negative GCLS had metachronous colorectal and endometrial cancers, colorectal and breast cancers, lung and uterine cervical cancers, lung cancer, and prostatic cancer, respectively. Two patients with EBV-positive GCLS had metachronous colorectal and lung cancers, and prostate cancer, respectively. Among them, two patients with EBV-negative GCLS and one with EBV-positive GCLS met the criteria under the revised Bethesda guidelines (Umar et al. 2004), but not under the revised Amsterdam criteria (Vasen et al. 1999). There were no significant between-group differences in multifocality, histologic type, tumour size, T category, perineural invasion, lymphatic or vascular invasion, $\mathrm{N}$-category, and pathologic AJCC stage except tumour location. 
Table 1

Clinicopathologic characteristics of patients with GCLS according to EBV infection status.

\begin{tabular}{|c|c|c|c|}
\hline & EBV negative & EBV positive & $P$ \\
\hline & GCLSs ( 30 cases) & GCLSs (74 cases) & \\
\hline Age (mean, range) & $65.5(32-82)$ & $55.6(31-77)$ & $<0.0001^{\star}$ \\
\hline$\leq 50$ years & $2(6.7 \%)$ & $25(33.8 \%)$ & $0.0056^{\star}$ \\
\hline$>50$ years & $28(93.3 \%)$ & $49(66.2 \%)$ & \\
\hline Sex & & & \\
\hline Male & $16(53.3 \%)$ & $69(93.2 \%)$ & $<0.0001 *$ \\
\hline Female & $14(46.7 \%)$ & $5(6.8 \%)$ & \\
\hline Other malignancy history except GAC & & & \\
\hline No & $25(83.3 \%)$ & $72(97.3 \%)$ & $0.0203^{*}$ \\
\hline Yes & $5(16.7 \%)$ & $2(2.7 \%)$ & \\
\hline History of metachronous cancers & & & \\
\hline Colorectal and endometrial cancers & $1(3.3 \%)$ & 0 & \\
\hline Colorectal and breast cancers & $1(3.3 \%)$ & 0 & \\
\hline Colorectal and lung cancers & 0 & $1(1.4 \%)$ & \\
\hline Lung and uterine cervical cancers & $1(3.3 \%)$ & 0 & \\
\hline Lung cancer & $1(3.3 \%)$ & 0 & \\
\hline Prostate cancer & $1(3.3 \%)$ & $1(1.4 \%)$ & \\
\hline Fulfillment of the rBG & & & \\
\hline No & $28(93.3 \%)$ & $73(98.6 \%)$ & 0.1991 \\
\hline Yes & $2(6.7 \%)$ & $1(1.4 \%)$ & \\
\hline Multifocality & & & \\
\hline Single & $28(93.3 \%)$ & $64(86.5 \%)$ & 0.5015 \\
\hline Multiple & $2(6.7 \%)$ & $10(13.5 \%)$ & \\
\hline Histologic type & & & \\
\hline Data are presented at $\mathrm{n}(\%)$ & & & \\
\hline $\begin{array}{l}\text { EBV, Epstein-Barr virus; GCLS, gastri } \\
\text { rBG, revised Bethesda Guidelines for } \\
\text { cancer; AGC, advanced gastric cance }\end{array}$ & $\begin{array}{l}\text { oma with lymphoid } \\
\text { syndrome and micrs }\end{array}$ & $\begin{array}{l}\text { ma; GAC, gastric ac } \\
\text { ellite instability; EG( }\end{array}$ & $\begin{array}{l}\text { carcinoma; } \\
\text { arly gastric }\end{array}$ \\
\hline
\end{tabular}




\begin{tabular}{|c|c|c|c|}
\hline & EBV negative & EBV positive & $P$ \\
\hline & GCLSs (30 cases) & GCLSs (74 cases) & \\
\hline EGC & $8(26.7 \%)$ & $25(33.8 \%)$ & 0.6424 \\
\hline AGC & $22(73.3 \%)$ & $49(66.2 \%)$ & \\
\hline \multicolumn{4}{|l|}{ Tumor location } \\
\hline Upper & $2(6.7 \%)$ & $14(18.9 \%)$ & $0.0001^{*}$ \\
\hline Middle & $15(50 \%)$ & $54(73 \%)$ & \\
\hline Lower & $13(43.3 \%)$ & $6(8.1 \%)$ & \\
\hline Tumor size (mean) & $3.69(1.4-11.5)$ & 3.85 (1.1-13.6) & 0.7616 \\
\hline \multicolumn{4}{|l|}{$\mathrm{T}$ category } \\
\hline $\mathrm{T} 1$ and $\mathrm{T} 2$ & $21(70 \%)$ & $45(60.8 \%)$ & 0.5009 \\
\hline T3 and T4 & $9(30 \%)$ & $29(39.2 \%)$ & \\
\hline \multicolumn{4}{|l|}{ Perineural invasion } \\
\hline Absent & 25 (83.3\%) & 57 (77\%) & 0.6002 \\
\hline Present & $5(16.7 \%)$ & 17 (30\%) & \\
\hline \multicolumn{4}{|l|}{ Lymphatic invasion } \\
\hline Absent & $17(56.7 \%)$ & $53(72.6 \%)$ & 0.1689 \\
\hline Present & $13(43.3 \%)$ & $21(28.4 \%)$ & \\
\hline \multicolumn{4}{|l|}{ Vascular invasion } \\
\hline Absent & $28(93.3 \%)$ & $62(83.8 \%)$ & 0.3409 \\
\hline Present & $2(6.7 \%)$ & $12(16.2 \%)$ & \\
\hline \multicolumn{4}{|l|}{$\mathrm{N}$ category } \\
\hline NO & $22(73.3 \%)$ & $48(64.9 \%)$ & 0.4303 \\
\hline N1 & $6(20 \%)$ & $14(18.9 \%)$ & \\
\hline N2 and N3 & $2(6.7 \%)$ & $12(16.2 \%)$ & \\
\hline AJCC stage & & & \\
\hline
\end{tabular}

Data are presented at $\mathrm{n}(\%)$

EBV, Epstein-Barr virus; GCLS, gastric carcinoma with lymphoid stroma; GAC, gastric adenocarcinoma; rBG, revised Bethesda Guidelines for Lynch syndrome and microsatellite instability; EGC, early gastric cancer; AGC, advanced gastric cancer 


\begin{tabular}{|llll|}
\hline & EBV negative & EBV positive & $P$ \\
\hline GCLSs (30 cases) & GCLSs (74 cases) & \\
\hline I and II & $27(90 \%)$ & $63(85.1 \%)$ & 0.7524 \\
\hline III and IV & $3(10 \%)$ & $11(14.9 \%)$ & \\
\hline
\end{tabular}

Data are presented at $\mathrm{n}(\%)$

EBV, Epstein-Barr virus; GCLS, gastric carcinoma with lymphoid stroma; GAC, gastric adenocarcinoma; $\mathrm{rBG}$, revised Bethesda Guidelines for Lynch syndrome and microsatellite instability; EGC, early gastric cancer; AGC, advanced gastric cancer

\section{Immunohistochemical results of MMR proteins and PD-L1, MSI status, and hypermethylation of MLH1}

Immunohistochemical analysis indicated that 15 (50\%) EBV-negative tumours showed loss of both MLH1 and PMS2 expression. In contrast, only one (1.4\%) EBV-positive tumour exhibited loss of both MLH1 and PMS2 expression. MSI testing confirmed that these 16 (15.4\%) samples were MSI-H tumours. However, there were no GCLS cases with loss of MSH2 and MSH6 expression. In 16 GCLS tumours with MSI-H and loss of MLH1 and PMS2 expression, MLH1 promoter methylation analysis revealed hypermethylation in 14 (87.5\%) cases, of which 13 (92.9\%) and 1 (7.1\%) were EBV-negative and EBVpositive tumours. Only two (12.5\%) EBV-negative GCLS tumours were negative for MLH1 hypermethylation. Among 104 GCLS tumours, 26 (25\%) were considered PD-L1 positive tumours with 2+ or $3+$ intensity and $\geq 10 \%$ of positive tumour cells. Among the 30 EBV-negative tumours, four $(13.3 \%)$ showed membranous and/or cytoplasmic staining patterns for PD-L1. Among the 74 EBV-positive tumours, 22 (29.7\%) exhibited PD-L1 positivity. While there was no significant between-group difference in the rates of PD-L1 positivity, EBV-positive tumours tended to have a higher rate of PD-L1 positivity than EBV-negative tumours $(13.3 \%$ vs. $29.7 \%, p=0.1321)$. The results of immunohistochemistry, MSI testing, and methylation analysis of EBV-negative and positive GCLS cases are presented in Table 2. 
Table 2

Immunohistochemical results for MMR proteins and PD-L1, MSI testing, and methylation analysis

EBV negative

EBV positive

$P$

GCLSs (30 cases)

GCLSs (74 cases)

MMR proteins

Intact

$15(50 \%)$

$73(98.6 \%)$

$<0.0001 *$

Loss of MLH1 and PMS2

$15(50 \%)$

$1(1.4 \%)$

MSI testing

MSS

$15(50 \%)$

$73(98.6 \%)$

$<0.0001^{*}$

MSI-H

$15(50 \%)$

$1(1.4 \%)$

MLH1-methylation analysis

(16 MSI-H tumors)

Unmethylated

$2(13.3 \%)$

0

$>0.9999$

MLH1 hypermethylated

$13(86.7 \%)$

$1(100 \%)$

PD-L1 positivity

Negative

$26(86.7 \%)$

$52(70.3 \%)$

0.1321

Positive

$4(13.3 \%)$

$22(29.7 \%)$

Proportion of PD-L1 positivity

$\geq 10 \%$

$1(25 \%)$

$8(36.4 \%)$

$>0.9999$

$\geq 50 \%$

$3(75 \%)$

$14(63.6 \%)$

MMR, mismatch repair; MSI, microsatellite instability; EBV, Epstein-Barr virus;MSS, microsatellite stable; MSI-H, microsatellite instability-high; PD-L1, programmed death-ligand 1

\section{Clinicopathologic features of MSI-H vs. MSS tumours and PD-L1 positive vs. PD-L1 negative tumours}

Comparisons of the clinicopathological features of MSI-H and MSS tumours are summarised in Table 3. Patients with MSI-H tumours were associated with older age, male sex, negative EBV infection status, and tumour occurrence in the distal stomach. However, there were no significant between-group differences in other clinicopathologic factors, such as the T- and N-category and AJCC stage. The clinicopathologic features of PD-L1 positive and negative tumours are presented in Table 4. Patients with PD-L1 positive tumours were associated with advanced gastric cancers ( $92.3 \%$ vs. $60.3 \%, p=0.0029)$, a higher $T$ category (T3 and T4, $57.5 \%$ vs. $29.5 \%, p=0.0175$ ) and a more advanced pathologic AJCC stage (III and IV, $26.9 \%$ vs. $9 \%, p=0.0407)$ compared with those of patients with PD-L1 negative tumours. 
Table 3

Comparison of clinicopathologic characteristics of patients with GCLS according to MSI status

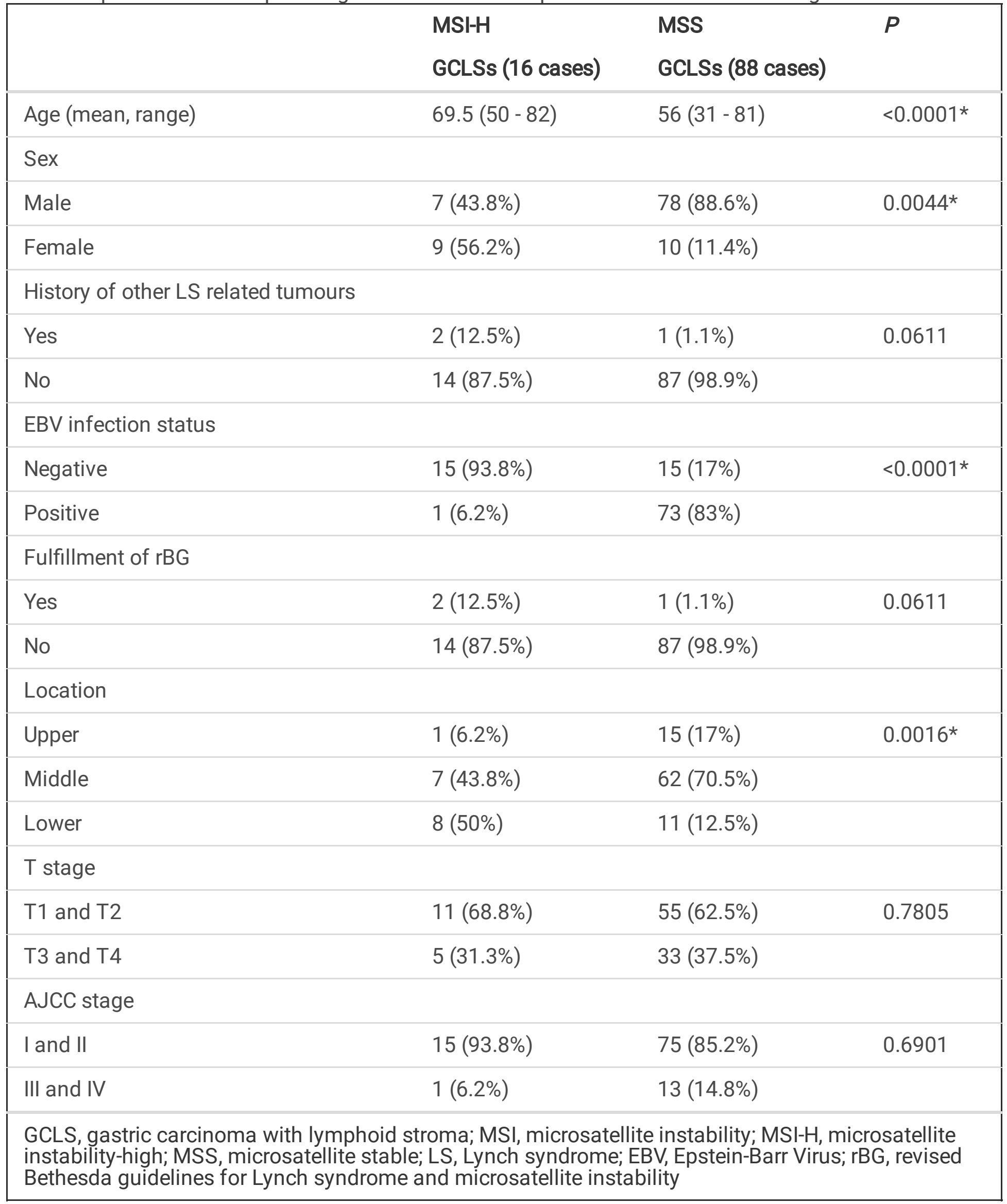


Table 4

Comparison of clinicopathologic characteristics of patients according to PD-L1 positivity

\begin{tabular}{|c|c|c|c|}
\hline & $\begin{array}{l}\text { PD-L1 positive } \\
\text { GCLSs ( } 26 \text { cases) }\end{array}$ & $\begin{array}{l}\text { PD-L1 negative } \\
\text { GCLSs (78 cases) }\end{array}$ & $P$ \\
\hline Age (mean, range) & $62(34-79)$ & $58(31-82)$ & 0.7065 \\
\hline \multicolumn{4}{|l|}{ Sex } \\
\hline Male & $21(80.8 \%)$ & $64(82.1 \%)$ & $>0.9999$ \\
\hline Female & $5(19.2 \%)$ & $14(17.9 \%)$ & \\
\hline \multicolumn{4}{|l|}{ EBV infection status } \\
\hline Negative & $4(15.4 \%)$ & $26(33.3 \%)$ & 0.1321 \\
\hline Positive & $22(84.6 \%)$ & $52(66.7 \%)$ & \\
\hline \multicolumn{4}{|l|}{ Histologic type } \\
\hline EGC & $2(7.7 \%)$ & $31(39.7 \%)$ & $0.0029 *$ \\
\hline AGC & $24(92.3 \%)$ & $47(60.3 \%)$ & \\
\hline \multicolumn{4}{|l|}{ Tumor location } \\
\hline Upper & $3(11.5 \%)$ & $13(16.7 \%)$ & 0.821 \\
\hline Middle & $18(69.2 \%)$ & $51(65.4 \%)$ & \\
\hline Lower & $5(19.2 \%)$ & $14(17.9 \%)$ & \\
\hline \multicolumn{4}{|l|}{ T category } \\
\hline $\mathrm{T} 1$ and $\mathrm{T} 2$ & $11(42.3 \%)$ & 55 (70.5\%) & $0.0175^{\star}$ \\
\hline T3 and T4 & $15(57.7 \%)$ & $23(29.5 \%)$ & \\
\hline \multicolumn{4}{|l|}{ AJCC stage } \\
\hline I and II & $19(73.1 \%)$ & $71(91 \%)$ & $0.0407 *$ \\
\hline III and IV & 7 (26.9\%) & $7(9 \%)$ & \\
\hline \multicolumn{4}{|l|}{ MSI status } \\
\hline MSS & $23(88.5 \%)$ & $65(83.3 \%)$ & 0.7553 \\
\hline MSI-H & $3(11.5 \%)$ & $13(16.7 \%)$ & \\
\hline
\end{tabular}

\section{Analysis of germline mutations}


WES was performed on formalin-fixed paraffin-embedded blocks of healthy tissue for seven patients suspicious of Lynch syndrome. Among them, two patients without EBV infection and MLH1 hypermethylation, and with loss of MLH1 and PMS2 expression, were diagnosed as having germline pathogenic variants. One patient had a history of colorectal and endometrial cancers and harboured PMS2 c. $1721 \mathrm{~A}>\mathrm{G}$ germline mutations, while the other had a history of colorectal and breast cancers and exhibited MLH1 X264 splice alteration.

\section{Survival}

Follow-up data were available for all 104 patients with GCLS. The 5- and 10-year OS rates for all 104 patients were $85.2 \%$ and $81.5 \%$, respectively, with a median follow-up of 126.5 months (range, 0.3 to 167 months).

Univariate Cox analysis identified three factors that were significantly associated with an improved median OS: T-category $(p=0.024), N$-category $(p=0.049)$, and pathologic AJCC stage $(p=0.012)$. Multivariate Cox analysis showed that the AJCC stage was the sole independent prognostic factor $(p=0.009)$ for these tumours. There were no significant statistical differences in OS rates between the EBV-positive and EBV-negative ( $p=0.281), M S I-H$ and MSS ( $p=0.493)$, and PD-L1 positive and negative tumour groups $(p=0.456)$.

\section{Discussion}

In the present study, patients with EBV-negative GCLS were more likely to be older and female, with a more significant personal history of cancers, and a primary tumour site predisposition to the distal stomach as the primary tumour site, compared to patients with EBV-positive GCLS. Due to the rarity of EBV-negative GCLS cases, only a few small studies exist regarding this type of tumours. Four prior studies reported that patients with EBV-negative GCLS were more significantly associated with older age, female sex, and a tumour location in the distal stomach than patients with EBV-positive GCLS (Min et al. 2016; Chang et al. 2000; Nakamura et al. 1994; Cho et al. 2018). Furthermore, Min et al. demonstrated that EBV-negative GCLS was significantly associated with a high T-category and an advanced AJCC tumour stage, resulting in a poorer OS than that of EBV-positive GCLS (Min et al. 2016). They also reported that EBV infection was an independent predictor of OS in patients with GCLS. However, in this series, apart from older age, personal history of cancers, female sex, and distal tumour location, there were no statistical differences in other clinicopathological features, such as T-category, N-category, AJCC stage, and survival differences between the EBV-negative and EBV-positive tumour groups. In the Cox proportional hazards model, AJCC tumour stage was observed to be the only independent predictor of OS. EBV infection, MSI status, and PD-L1 expression were not statistically correlated with OS in GCLS tumours. The discrepancies between the two Asian studies may be caused by selection biases due to the small number of studied cases. Large-scale studies on EBV-negative GCLS tumours are required to verify whether EBV infection serves as a pivotal prognostic indicator for OS in patients with GCLS. 
MSI-H tumours were reportedly observed in $22 \%$ of GCs in the Western population and $6.8 \%-10.3 \%$ in the Asian population (Choi et al. 2019; Cho et al. 2019; Cancer Genome Atlast Research Network 2014). The prevalence of the MSI-H phenotype may be affected by the different methods used to detect MSI, such as immunohistochemistry for MMR proteins and MSI testing (Mathiak et al. 2017). Furthermore, racial or geographic differences and histologic subtypes of GC may affect the incidence of the MSI-H phenotype in GCs. Among the histologic subtypes of GC, our study demonstrated that EBV-negative GCLSs were associated with a higher prevalence of MSI-H than that associated with EBV-positive GCLSs $(50 \%$ vs. $1.4 \%, \mathrm{p}<0.0001)$ and even compared with those reported for conventional GCs from an Asian data (50\% vs. $10.3 \%, p<0.0001$ ) (Choi et al. 2019). Similarly, Min et al. (2016) performed MSI testing and reported a relatively high frequency of MSI-H associated with EBV-negative GCLS (25\%,5/25 cases). Based on the results of the two studies, the MSI-H phenotype tends to be more frequently observed in EBV-negative GCLS tumours. However, the predominant histologic type of MSI-H tumour is the intestinal GC, followed by non-intestinal types due to relatively low occurrences of non-intestinal histologic subtypes (Choi et al. 2019). Cristescue et al. (2015) reported that EBV infection in GCs was correlated with a predominantly MSS status, mutually exclusive with the MSI-H phenotype; similarly, using immunohistochemistry and MSI testing, we found that approximately $99 \%$ of EBV-positive GCLS were MSS tumours. Regarding the expression of MMR proteins and MLH1 hypermethylation in GCs, lto et al. reported that $93 \%$ of GCs with MSI-H exhibited loss of MLH1 and/or PMS2 expression by immunohistochemistry (Ito et al. 2021). Approximately $95 \%$ of GCs with MSI-H showed hypermethylation of the promotor region of MLH1 (Ito et al. 2021). Similarly, in our study, all $16(100 \%)$ cases of GCLS with MSI-H showed loss of expression of the MLH1 and PMS2 proteins. Methylation analysis of $16 \mathrm{MSI}-\mathrm{H}$ tumours revealed hypermethylation of MLH1 in $87.5 \%$ (14/16) of GCLS cases. Previous studies have demonstrated that hypermethylation of MLH1 occurs in approximately $77.8 \%-100 \%$ of GCs with MSI-H, indicating that our findings are within the range of previous data (Ito et al. 2021; Fleisher et al. 1999; Sakata et al. 2002).

It is estimated that $2 \%-4 \%$ of CRC and $1.8 \%-5.2 \%$ of upper urinary tract urothelial cancer cases are attributed to LS (Ito et al. 2020; Cunningham et al. 2001; Hampel et al. 2005; Barnetson et al. 2006; Ikenoue et al. 2019; Metcalfe et al. 2018). The prevalence of LS in unselected patients with GC has not been well investigated. Although the incidence of GC is high in Japan and South Korea, evaluation of genetic factors such as LS in patients with GC was only recently initiated. In a study of 513 consecutive patients with primary GC, Ito et al. reported that $0.2 \%(1 / 513)$ of patients with GC and $1.7 \%(1 / 58)$ of GC patients with MSI-H were found to have LS (Ito et al. 2021). In our study, we identified LS in $1.9 \%(2 / 104)$ of patients with GCLS and in $12.5 \%(2 / 16)$ of patients with GCLS with the MSI-H phenotype. Following immunohistochemistry for MMR proteins and MSI testing, we evaluated the personal and family history of cancers in patients with MSI-H tumours. Three patients fulfilled the revised Bethesda criteria, including LS-related cancers. After methylation analysis for MLH1, one patient was found to have an MLH-1 hypermethylated tumour. After performing WES analysis, two (6.7\%) patients with MSI-H GCLS and a history of LS-related cancers were found to have germline mutations in MLH1 and PMS2. Based on our findings, in cases of tumours with the morphological features of GCLS according to the WHO diagnostic criteria, EBV infection status should be investigated, along with MSI analysis. Depending on the MSI 
status of GCLS, recommendations should be made for MLH1 methylation analysis, review of personal history of cancer, and genetic testing for the detection of LS in patients with GCLS.

Recently, the US Food and Drug Administration (FDA) granted pembrolizumab, a PD-1 inhibitor, approval for the treatment of metastatic solid tumours with deficient DNA mismatch repair (dMMR) or with MSI-H, regardless of tumour type (Marcus et al. 2019). The US FDA also approved PD-L1 immunohistochemistry assays as companion diagnostic tests for the corresponding drugs for cases of non-small lung cancer, because the drugs were more effective for PD-L1 positive tumours (US Food and Drug Administration 2016 and 2017; Roche 2021). Choi et al. investigated MSI and PD-L1 expression in 592 cases of stage II/III GC. They reported that $40(6.8 \%)$ of 592 tumours were of the MSI-H phenotype and $16(2.7 \%)$ of 582 tumours showed PD-L1 positivity with scores of $2+$ and $3+$ and $\geq 10 \%$ of positive tumour cells (Choi et al. 2019). In the present study, $26(25 \%)$ of 104 GCLS tumours showed PD-L1 positivity, with scores of 2+ and $3+$ and $\geq 10 \%$ of positive tumour cells. The rate of PD-L1 positivity in GCLS tumours was higher than that previously reported for conventional GCs ( $25 \%$ vs. $2.7 \%, \mathrm{p}<0.0001)$ (Choi et al. 2019). EBV-positive GCLS tumours were associated with a slightly higher rate of PD-L1 positivity than EBV-negative tumours (13.3\% vs. $29.7 \%, p=0.1321)$. Our data suggest that patients with advanced GCLS, regardless of EBV infection, can be good candidate for immunotherapy including PD-1 and PD-L1 inhibitors.

The current study has some limitations. The differences in clinicopathologic features between observed in previous studies and this study may originate from selection biases due to the rarity of this tumour, and because this was a relatively small study conducted at a single institution. In addition, the survival data of GCLS may be limited due to the small number of associated deaths.

In summary, approximately $50 \%$ of EBV-negative GCLS tumours were MSI-H tumours showing loss of MLH1 and PMS expression. The prevalence of LS was estimated to be 1.9\% (2/104) among patients with GCLS and 6.7\% (2/30) among those with EBV-negative GCLS. Therefore, in cases of EBV-negative GCLS, evaluation of MSI and selective genetic testing are highly recommended for the detection of LS. In addition, patients with GCLS regardless of EBV infection are more likely to be good candidates for treatment with immune checkpoint inhibitors as compared to patients with conventional GC.

\section{Declarations}

Funding This work was supported by a research grant from Jeju National University Hospital in 2019.

Data availability All data generated that are relevant to the results presented in this article are included. Other data that were not relevant for the results presented are available from the corresponding author (SM Lee) upon reasonable request.

Conflicts of interest The authors report no potential conflicts of interest relevant to this article.

Ethical approval The study was conducted according to the guidelines of the Declaration of Helsinki and approved by the Institutional Review Board of Jeju National University Hospital. 


\section{References}

1. Kuiper RP, Vissers LE, Venkatachalam R, et al. (2011) Recurrence and variability of germline EPCAM deletions in Lynch syndrome. Hum Mutat. 32:407-414. https://doi.org/10.1002/humu.21446

2. Lynch HT, de la Chapelle A. (1999) Genetic susceptibility to non-polyposis colorectal cancer. J Med Genet. 36:801-18.

3. Bonadona V, Bonaiti B, Olschwang S, et al. (2011) Cancer risks associated with germline mutations in MLH1, MSH2, and MSH6 genes in Lynch syndrome. JAMA. 305:2304-2310. https://doi.org/10.1001/jama.2011.743

4. Watson P, Vasen HA, Mecklin JP, et al. (2008) The risk of extra-colonic, extra-endometrial cancer in the Lynch syndrome. Int J Cancer. 123:444-449. https://doi.org/10.1002/ijc.23508

5. Julie C, Tresallet C, Brouquet A, et al. (2008) Identification in daily practice of patients with Lynch syndrome (hereditary nonpolyposis colorectal cancer): revised Bethesda guidelines-based approach versus molecular screening. Am J Gastroenterol. 103:2825-2835. https://doi.org/10.1111/j.15720241.2008.02084.x

6. Stoffel EM, Mangu PB, Gruber SB, et al. (2015) Hereditary colorectal cancer syndromes: American Society of Clinical Oncology Clinical Practice Guideline endorsement of the familial risk-colorectal cancer: European Society for Medical Oncology Clinical Practice Guidelines. J Clin Oncol. 33:209217. https://doi.org/10.1200/JCO.2014.58.1322.

7. Raut CP, Pawlik TM, Rodriguez-Bigas MA. (2004) Clinicopathologic features in colorectal cancer patients with microsatellite instability. Mutat Res. 568:275-282.

https://doi.org/10.1016/j.mrfmmm.2004.05.025

8. Carneiro F, Fukayama M, Grabsch HI, et al. (2019) Gastric adenocarcinoma. In: WHO classification of tumours editor board (ed) WHO classification of tumours: digestive system tumours, 5th edn. Lyon: International Agency for Research on Cancer, Lyon, pp 91

9. Murphy G, Pfeiffer R, Camargo MC, et al. (2009) Meta-analysis shows that prevalence of Epstein-Barr virus-positive gastric cancer differs based on sex and anatomic location. Gastroenterology. 137:824833. https://doi.org/10.1053/j.gastro.2009.05.001

10. Shinozaki-Ushiku A, Kunita A, Fukayama M. (2015) Update on Epstein-Barr virus and gastric cancer (review). Int J Oncol. 46:1421-1434. https://doi.org/10.3892/ijo.2015.2856

11. Min BH, Tae CH, Ahn SM, et al. (2016) Epstein-Barr virus infection serves as an independent predictor of survival in patients with lymphoepithelioma-like gastric carcinoma. Gastric Cancer. 19:852-859. https://doi.org/10.1007/s10120-015-0524-x

12. Lee JH, Kim SH, Han SH, et al. (2009) Clinicopathological and molecular characteristics of EpsteinBarr virus-associated gastric carcinoma: a meta-analysis. J Gastroenterol Hepatol. 24:354-365. https://doi.org/10.1111/j.1440-1746.2009.05775.x

13. Camargo MC, Kim WH, Chiaravalli AM, et al. (2014) Improved survival of gastric cancer with tumour Epstein-Barr virus positivity: an international pooled analysis. Gut. 63:236-243. 
https://doi.org/10.1136/gutjnl-2013-304531

14. Park S, Choi MG, Kim KM, et al. (2015) Lymphoepithelioma-like carcinoma: a distinct type of gastric cancer. J Surg Res. 194:458-463. https://doi.org/10.1016/j.jss.2014.12.005

15. Saito M, Kono K. (2021) Landscape of EBV-positive gastric cancer. Gastric Cancer. 24:983-989. https://doi.org/10.1007/s10120-021-01215-3

16. Ajani JA, In HJ, Sano T, et al (2017) Stomach. In: Amin MB (ed) AJCC cancer staging manual, 8th edn. Springer Nature Publisher, Chicago, pp 203-220.

17. Umar A, Boland CR, Terdiman JP, et al. (2004) Revised bethesda guidelines for hereditary nonpolyposis colorectal cancer (Lynch syndrome) and microsatellite instability. J Natl Cancer Inst. 96:261-268. https://doi.org/10.1093/jnci/djh034

18. Vasen HF, Watson P, Mecklin JP, et al. (1999) New clinical criteria for hereditary nonpolyposis colorectal cancer (HNPCC, Lynch syndrome) proposed by the International Collaborative group on HNPCC. Gastroenterology. 116:1453-1456. https://doi.org/10.1016/s0016-5085(99)70510-x.

19. Phillips T, Simmons P, Inzunza HD, et al. (2015) Development of an automated PD-L1 immunohistochemistry (IHC) assay for non-small cell lung cancer. Appl Immunohistochem Mol Morphol. 23:541-549. https://doi.org/10.1097/PAl.0000000000000256

20. Choi YY, Kim H, Shin SJ, et al. (2019) Microsatellite instability and programmed cell death-ligand 1 expression in stage II/III gastric cancer: post hoc analysis of the CLASSIC randomized controlled study. Ann Surg. 270:309-316. https://doi.org/10.1097/SLA.0000000000002803

21. Laiho P, Launonen V, Lahermo P, et al. (2002) Low-level microsatellite instability in most colorectal carcinomas. Cancer Res. 62:1166-1170.

22. Ogino S, Kawasaki T, Brahmandam M, et al. (2006) Precision and performance characteristics of bisulfite conversion and real-time PCR (MethyLight) for quantitative DNA methylation analysis. J Mol Diagn. 8:209-217. https://doi.org/10.2353/jmoldx.2006.050135

23. Chang MS, Kim WH, Kim CW, et al. (2000) Epstein-Barr virus in gastric carcinomas with lymphoid stroma. Histopathology. 37:309-315. https://doi.org/10.1046/j.1365-2559.2000.01014.x

24. Nakamura S, Ueki T, Yao T, et al. (1994) Epstein-Barr virus in gastric carcinoma with lymphoid stroma. Special reference to its detection by the polymerase chain reaction and in situ hybridization in 99 tumors, including a morphologic analysis. Cancer. 73:2239-2249. https://doi.org/10.1002/1097-0142(19940501)73:9<2239::aid-cncr2820730902>3.0.co;2-\#

25. Cho CJ, Kang HJ, Ryu YM, et al. (2018) Poor prognosis in Epstein-Barr virus-negative gastric cancer with lymphoid stroma is associated with immune phenotype. Gastric Cancer. 21:925-935. https://doi.org/10.1007/s10120-018-0820-3

26. Cho J, Kang SY, Kim KM. (2019) MMR protein immunohistochemistry and microsatellite instability in gastric cancers. Pathology. 51:110-113. https://doi.org/10.1016/j.pathol.2018.09.057

27. Cancer Genome Atlas Research Network (2014) Comprehensive molecular characterization of gastric adenocarcinoma. Nature. 513:202-209. https://doi.org/10.1038/nature13480 
28. Mathiak M, Warneke VS, Behrens HM, et al. (2017) Clinicopathologic characteristics of microsatellite instable gastric carcinomas revisited: Urgent Need for Standardization. Appl Immunohistochem Mol Morphol. 25:12-24. https://doi.org/10.1097/PAl.0000000000000264

29. Cristescu R, Lee J, Nebozhyn M, et al. (2015) Molecular analysis of gastric cancer identifies subtypes associated with distinct clinical outcomes. Nat Med. 21:449-456. https://doi.org/10.1038/nm.3850

30. Ito T, Suzuki O, Kamae N, et al. (2021) Comprehensive analysis of DNA mismatch repair-deficient gastric cancer in a Japanese hospital-based population. Jpn J Clin Oncol. 51:886-894. https://doi.org/10.1093/jjco/hyab026

31. Fleisher AS, Esteller M, Wang S, et al. (1999) Hypermethylation of the hMLH1 gene promoter in human gastric cancers with microsatellite instability. Cancer Res. 59:1090-1095.

32. Sakata K, Tamura G, Endoh Y, et al. (2002) Hypermethylation of the hMLH1 gene promoter in solitary and multiple gastric cancers with microsatellite instability. $\mathrm{Br} \mathrm{J}$ Cancer. 86:564-567. https://doi.org/10.1038/sj.bjc.6600076

33. Ito T, Kono K, Eguchi H, et al. (2020) Prevalence of Lynch syndrome among patients with upper urinary tract carcinoma in a Japanese hospital-based population. Jpn J Clin Oncol. 50:80-88. https://doi.org/10.1093/jjco/hyz140

34. Cunningham JM, Kim CY, Christensen ER, et al (2001) The frequency of hereditary defective mismatch repair in a prospective series of unselected colorectal carcinomas. Am J Hum Genet. 69:780-790. https://doi.org/10.1086/323658

35. Hampel H, Frankel WL, Martin E, et al. (2005) Screening for the Lynch syndrome (hereditary nonpolyposis colorectal cancer). N Engl J Med. 352:1851-1860.

https://doi.org/10.1056/NEJMoa043146

36. Barnetson RA, Tenesa A, Farrington SM, et al. (2006) Identification and survival of carriers of mutations in DNA mismatch-repair genes in colon cancer. N Engl J Med. 354:2751-2763. https://doi.org/10.1056/NEJMoa053493

37. Ikenoue T, Arai M, Ishioka C, et al. (2019) Importance of gastric cancer for the diagnosis and surveillance of Japanese Lynch syndrome patients. J Hum Genet. 64:1187-1194. https://doi.org/10.1038/s10038-019-0674-5

38. Metcalfe MJ, Petros FG, Rao P, et al. (2018) Universal point of care testing for lynch syndrome in patients with upper tract urothelial carcinoma. J Urol. 199:60-65. https://doi.org/10.1016/j.juro.2017.08.002

39. Marcus L, Lemery SJ, Keegan P, et al. (2019) FDA approval summary: pembrolizumab for the treatment of microsatellite instability-high solid tumors. Clin Cancer Res. 25: 3753-3758. https://doi.org/10.1158/1078-0432.CCR-18-4070

40. US Food and Drug Administration (2016) Pembrolizumab (KEYTRUDA) checkpoint inhibitor. https://www.fda.gov/drugs/resources-information-approved-drugs/pembrolizumab-keytrudacheckpoint-inhibitor. Assessed 24 October 2016 
41. US Food and Drug Administration (FDA) (2017) FDA grants nivolumab accelerated approval for MSI$\mathrm{H}$ or dMMR colorectal cancer. https://www.fda.gov/drugs/resources-information-approveddrugs/fda-grants-nivolumab-accelerated-approval-msi-h-or-dmmr-colorectal-cancer. Assessed 31 July 2017

42. Roche (2021) Roche's Ventana PD-L1 (SP263) assay receives FDA approval as a companion diagnostic to identify certain non-small cell lung cancer patients eligible for Tecentriq ${ }^{\circledR}($ atezolizumab). https://www.roche.com/media/releases/med-cor-2021-10-22.htm. Assessed 22 October 2021.

\section{Figures}

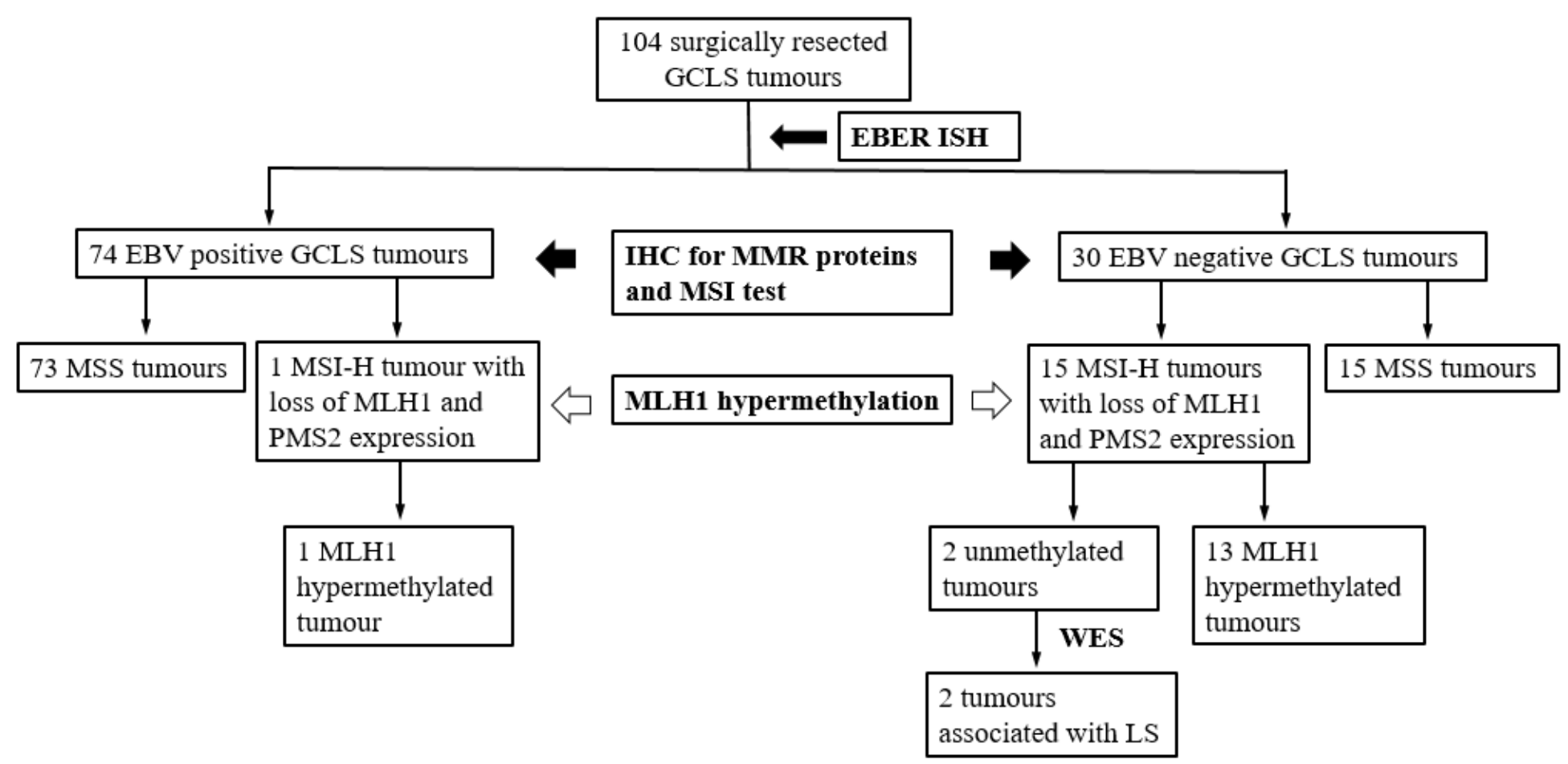

\section{Figure 1}

Flowchart of stepwise screening tests for microsatellite instability (MSI) and Lynch syndrome (LS) by immunohistochemistry (IHC) for mismatch repair (MMR) proteins, MSI testing and whole exome sequencing (WES).

GCLS, gastric carcinoma with lymphoid stroma; EBER ISH, Epstein-Barr encoding region in situ hybridization; EBV, Epstein-Barr virus; MSS, microsatellite stable; MSI-H, microsatellite instability-high. 
Histopathologic and immunohistochemical images of Epstein-Barr virus (EBV) negative gastric carcinoma with stroma (GCLS). The undifferentiated or poorly differentiated carcinoma with syncytial growth pattern is densely infiltrated by mature small lymphocytes on a hematoxylin \& eosin stain (a). The carcinoma component shows complete loss of MLH1 (b) and PMS2 (c) expression. The tumor cells exhibit strong membranous and/or cytoplasmic staining pattern of PD-L1 (d). Original magnification: $a, b$, c \& d, 40x.
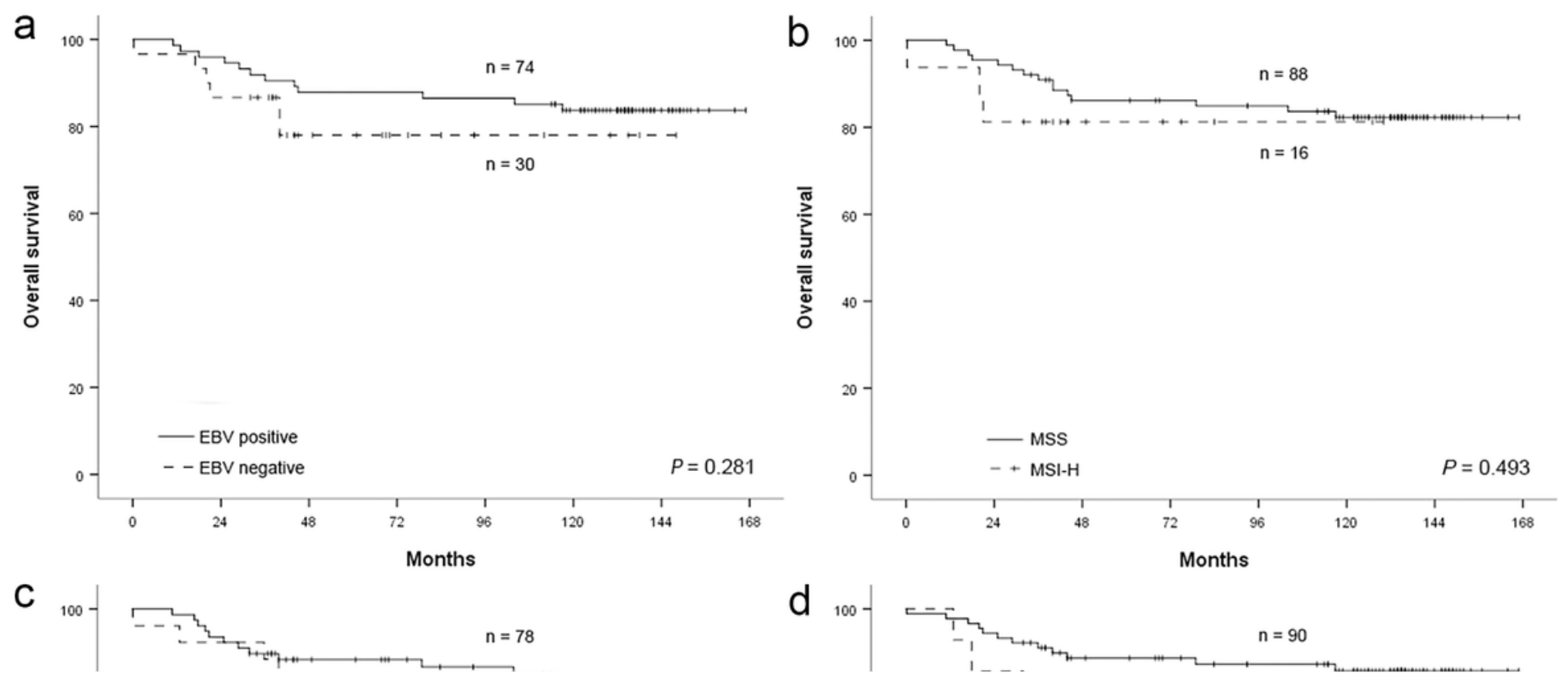

\section{Figure 3}

Kaplan-Meier cumulative survival curves according to EBV infection, MSI status, PD-L1 positivity and AJCC stage. EBV infection, negative vs. positive tumors (a). MSI status, MSI-H vs. MSS tumors (b). PD-L1 negative vs. PD-L1 positive groups (c). AJCC stage, I and II vs. III and IV stage (d). Only median survival for AJCC stage was statistically significant $(p=0.006)$. 